\title{
Three-dimensional medical image recognition of the cancer of the liver by artificial neural network
}

\author{
Masahiro Nakagawa, Tadashi Kondo ${ }^{1)}$, Tsuyosi Kudo, \\ Shoichiro Takao and Junji Ueno \\ Graduate school of Health Sciences, The University of Tokushima \\ 3-18-15 Kuramoto-cho, Tokushima 770-8509, Japan \\ E-mail: kondo@medsci.tokushima-u.ac.jp ${ }^{1)}$
}

\begin{abstract}
In this study, the three-dimensional medical image of the cancer of the liver, which is called Hepate-Cellular Carcinoma (HCC), is recognized and extracted by artificial neural network trained using the back propagation algorithm. First, the neural network recognizes the liver regions and the candidate regions of HCC. Then, the density difference image between the early phase image and late phase image of MDCT is extracted. The regions of $\mathrm{HCC}$ are detected using the density difference image and the candidate regions of HCC. These image processing are carried out for all slices of MDCT and three-dimensional images of HCC is displayed clearly.
\end{abstract}

\section{Introduction}

Recently, the number of slices scanned by multi detector raw computed tomography (MDCT) is increasing according to the development of medical imaging technology, and the number of images for the diagnosis becomes large and the doctor's burden is increasing. The computed aided diagnosis (CAD) is expected to reduce the doctor's diagnosis works and to improve diagnosis speed and accuracy. In this study, we apply the neural network to the CAD of the liver cancer which is called HCC.

The neural network used in this paper is the conventional sigmoid function type neural network trained using back propagation algorithm [1][2]. It has the three layered architecture that is constructed with the input layer, the hidden layer and the output layer. Weighs of the neural network are estimated using the back propagation algorithm. In this study, MDCT images are used. Ten input variables, which are four statistical image features, such as mean, variance, standard deviation and range, and four texture features and the two coordinates ( $x$ and $y$ ) of the neighboring regions are used. The neural network is applied to 3-dimensional medical image recognition of the liver regions and the candidate regions of the HCC are extracted. Then, the density difference image between the early phase image and the late phase image of MDCT is extracted. The regions of HCC are extracted using the density difference image and the candidate regions of HCC.
These image processing are carried out for all slices of MDCT and 3-dimentionl images of HCC is clearly displayed with the volume rendering software. Fig. 1 shows the flowchart of the extraction of the HCC regions.

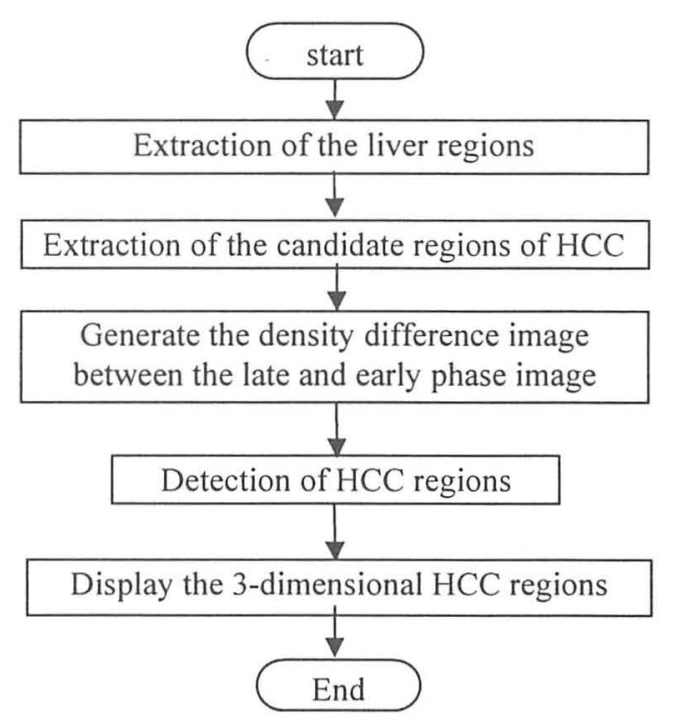

Fig.1 Flowchart of the extraction of the HCC regions

\section{Sigmoid function type neural network trained by back propagation algorithm}

The neural network using sigmoid function is organized with three layered architecture, which are the input, hidden and output layers, and its weights are trained using back propagation algorithm. Architecture of the neural network is shown in Fig.2. 


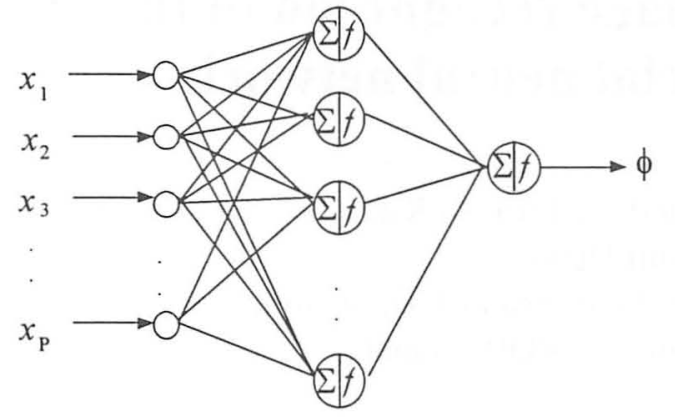

Fig.2 Architecture of the neural network trained using back propagation algorithm

\section{(1) Input layer}

$$
u_{i}=x_{i} \quad(i=1,2, \ldots, p)
$$

Here, $x_{j}$ is input variables and $p$ is the number of the input variables.

\section{(2) Hidden layer}

$$
\begin{aligned}
& y_{j}=\sum_{i}^{P} w_{i} u_{i}-\theta, \quad(j=1,2, \cdots, g) \\
& u_{j}=\frac{1}{1+\exp \left(-y_{j}\right)}, \quad(j=1,2, \cdots, g)
\end{aligned}
$$

Here, $w_{i}(i=1,2, \ldots, p)$ is the weights between the input and hidden layer and $g$ is the number of neurons in the hidden layer.

\section{(3) Output layer}

$$
\begin{aligned}
& y_{j}=\sum_{i}^{g} w_{i} u_{i}-\theta, \quad(j=1,2, \cdots r) \\
& u_{j}=\frac{1}{1+\exp \left(-y_{j}\right)}, \quad(j=1,2, \cdots r)
\end{aligned}
$$

Here, $w_{i}(i=1,2, \ldots, g)$ is the weights between the hidden and output layer and $r$ is the number of neurons in the output layer.

In this study, weights of artificial neural network are learned using the back propagation (BP) algorithm shown as follows:

$$
\begin{gathered}
\Delta w^{k-1}{ }_{j}{ }^{k}{ }_{i}=-\varepsilon \quad d^{k}{ }_{i} u^{k-1}{ }_{j} \\
d^{k}{ }_{i}=\left\{\begin{array}{lc}
\left(u^{m}{ }_{i}-\phi_{i}\right) u^{m}{ }_{i}\left(1-u^{m}{ }_{i}\right) & \text { for } k=m \\
\left.\left(\sum_{j} w^{k}{ }_{i}{ }^{k+1}{ }_{j} d^{k+1}\right)\right) u^{k}{ }_{i}\left(1-u^{k}{ }_{i}\right) & \text { for } k \neq m
\end{array}\right.
\end{gathered}
$$

Here, $w^{k}{ }_{i}^{k+1}$ is the weight between the $i$-th neuron in the $k$-th layer and the $j$-th neuron in the $k+1$-th layer. $\varepsilon$ is a coefficient. $u_{i}^{k}$ is output variable of the $i$-th neuron in the $k$-th layer. $m$ is the number of the output layer. $\phi_{i}$ is the $i$-th output variable of the system

\section{The input variables of the neural network}

In the neural network, ten input variables, which are four statistical image features, such as mean, variance, standard deviation and range, and four texture features [3] and two coordinates ( $x$ and $y$ ) of the neighboring region are used. The four texture features are calculated with a co-occurrence matrix. Co-occurrence matrix is constructed with probability $P_{\delta}(i, j),(i, j=0,1, \ldots, n-1)$ in the $n \times n$ neighboring region using a parameter $\delta=(r, \theta)$. Here, $r$ is a distance between gray level $i$ and gray level $j$ and $\theta$ is an angle. Four texture features are calculated using the co-occurrence matrix as follows;

Angular second moment:

$$
A S M=\sum_{i=0}^{n-1} \sum_{j=0}^{n-1}\left\{P_{\delta}(i, j)\right\}^{2}
$$

Contrast:

$$
C O N=\sum_{k=0}^{n-1} k^{2} \cdot p_{x-y}(k), \quad P_{x-y}(k)=\sum_{\substack{i=0 \\|i-j|=k}}^{n-1} \sum_{\substack{n-1 \\ i=0}}^{n-1} P_{\delta}(i, j) \quad k=0,1, \ldots, n-1
$$

Entropy:

$$
E N T=-\sum_{i=0}^{n-1} \sum_{j=0}^{n-1} P_{\delta}(i, j) \cdot \log \left\{P_{\delta}(i, j)\right\}
$$

Inverse difference moment:

$$
I D M=\sum_{i=0}^{n-1} \sum_{j=0}^{n-1} \frac{1}{1+(i-j)^{2}} P_{\delta}(i, j)
$$

\section{Application to medical image recognition of $\mathrm{HCC}$}

The neural network trained using back propagation algorithm was applied to the medical image recognition of HCC. MDCT images of the liver in the early and late phase were used in this study.

\subsection{Extraction of the liver regions}

An original image shown in Fig.3 was used for organizing the artificial neural networks. This image is an early phase image of MDCT. The initial values of the weights were set to random values. The learning calculations of the weights were iterated changing structural parameters such as the number of neurons in the hidden layer and the initial values of the weights. In this study, the number of neurons in the hidden layer was set to 
five. Ten input variables, which are four statistical image features and four texture features and the two coordinates ( $x$ and $y$ ) of the $\mathrm{N} \times \mathrm{N}$ regions were used. The output value of the neural network is zero or one. When $\mathrm{N} \times \mathrm{N}$ pixel region is contained in the region of the liver, the neural network sets the pixel value at the center of the $\mathrm{N} \times \mathrm{N}$ pixel region to one and this pixel is show as the white point. In this study, we set the value of $\mathrm{N}$ to 3 . Figure 4 shows the output image of the neural network. The post-processing analysis was carried out so as to eliminate the small isolate regions inside and outside of the liver. In the post-processing, small isolated regions were eliminated by the image processing such as the dilatation and the erosion. Then, outline of the liver regions were expanded outside by N/2 pixels and the outline of the livers was extracted. Figure 5 shows the output image after the post processing.

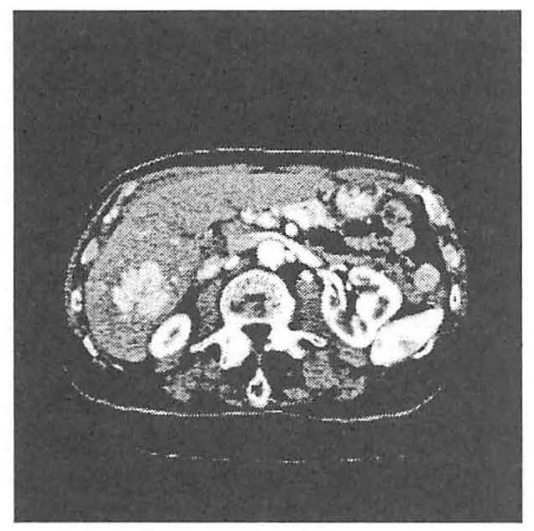

Fig.3 Original image obtained in the early phase

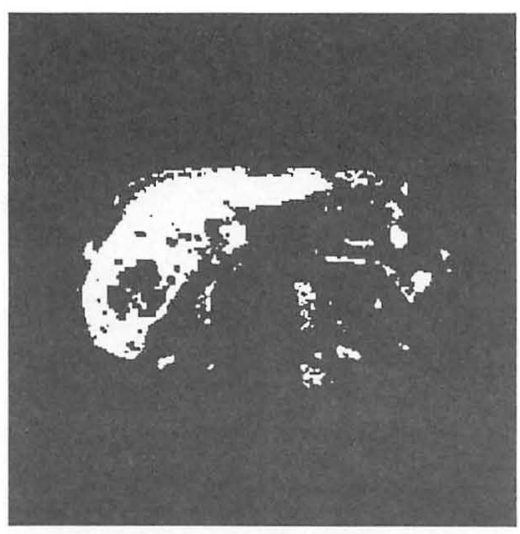

Fig.4 Output image of the neural network

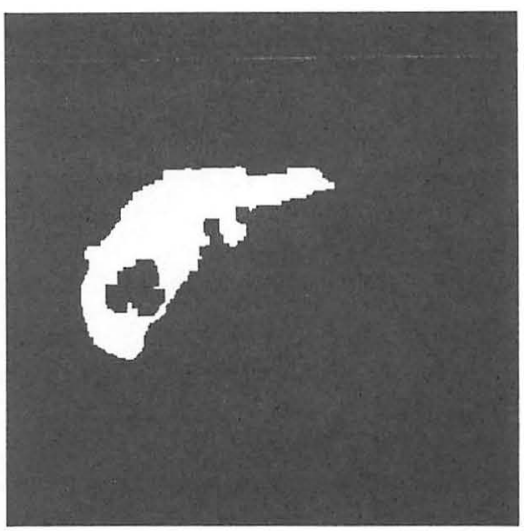

Fig.5 Output image after the post processing

\subsection{Extraction of the HCC}

The post-processing such as the dilatation and erosion was carried out so as to eliminate the isolate regions in the liver. Figure 6 shows the output image of the liver regions including the abnormal regions and the blood vessels in the liver. Then the candidate regions of the HCC, which contained the abnormal regions and the blood vessels in the liver, were subtracted from the liver regions using the output image of the neural network after the post-processing. Figure 7 shows the candidate regions of HCC. Figure 8 shows the late phase image of MDCT. In the late phase image of MDCT, the densities of HCC regions are lower than those of liver regions. In the early phase image (Fig.3) of MDCT, the densities of HCC regions are higher than those of liver regions. Therefore, the density difference image between the early and late phase image contain the HCC regions. The density difference image between the early phase image (Fig.3) and the late phase image (Fig.8) of MDCT was subtracted as shown in Fig.9 and the threshold processing was carried out to obtain the binary image. Figure 10 shows the density difference image after the threshold processing.

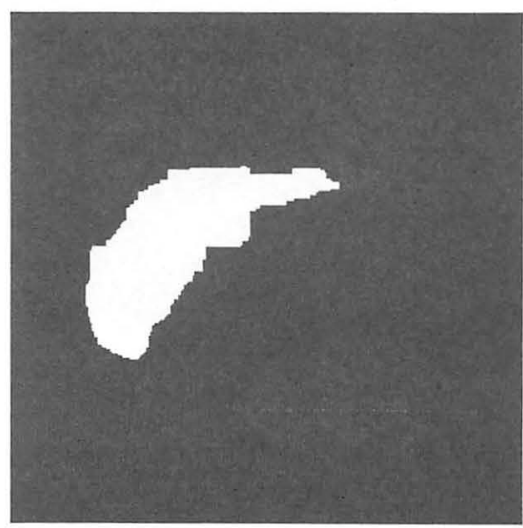

Fig.6 The liver region including the abnormal and the blood vessel regions in the liver 


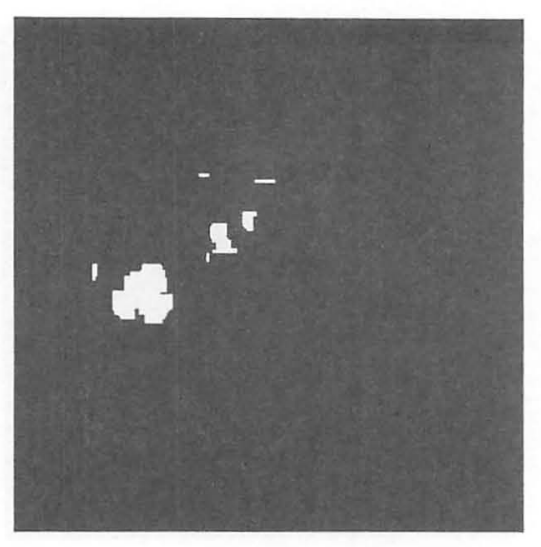

Fig.7 The candidate regions of $\mathrm{HCC}$

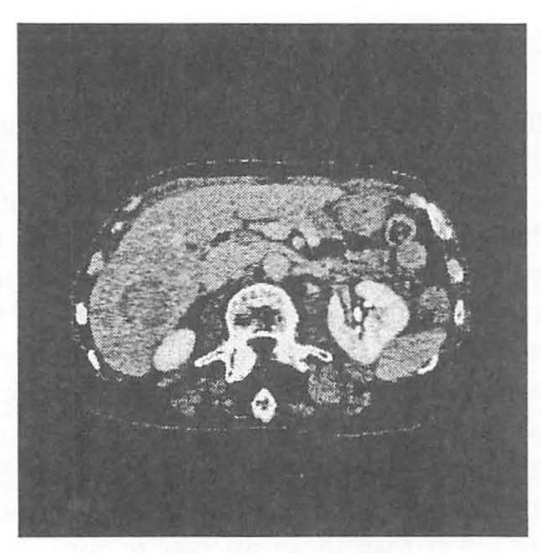

Fig.8 Original image obtained in the late phase

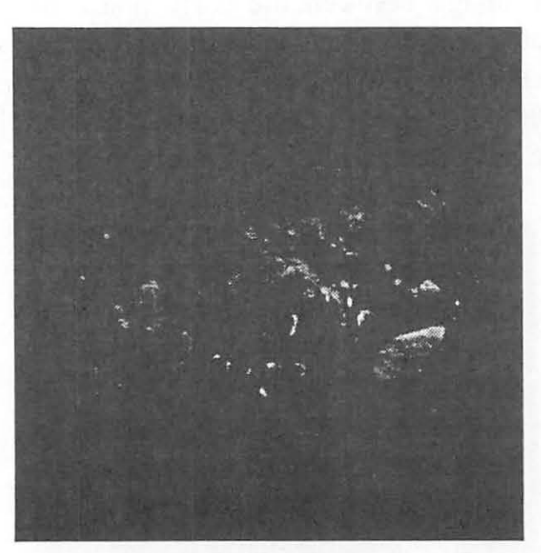

Fig.9 The density difference image

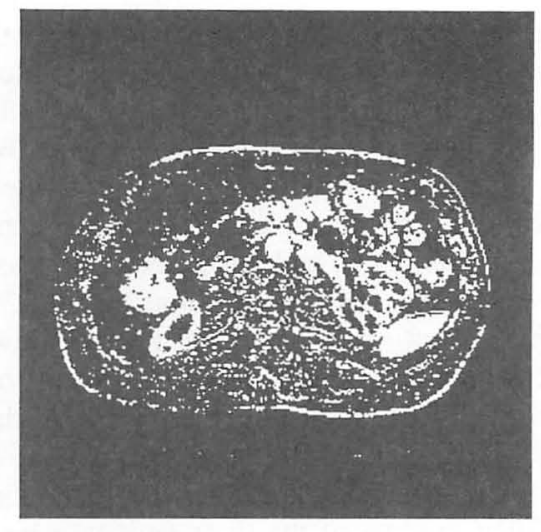

Fig.10 The density difference image after the threshold processing

The candidate regions of $\mathrm{HCC}$ shown in Fig.7 contain the low density regions in the liver. Besides, the density difference image after the threshold processing shown in Fig.10 contains the regions outside the liver and noise. $\mathrm{HCC}$ regions were detected as the common regions between the candidate regions of HCC (Fig.7) and the density difference image after the threshold processing (Fig.10). Figure 11 shows the extracted region of HCC. The post processing analysis was carried out so as to eliminate the small isolated regions such as the blood vessels in the liver and noise. In the post processing, small isolated regions were eliminated by the image processing such as the circumference length processing and filtering. Fig. 12 shows the HCC regions after the post processing.

These image processing were carried out for all slices of MDCT images and 3-dimentional images of HCC are displayed with volume rendering software as shown in Fig.13.

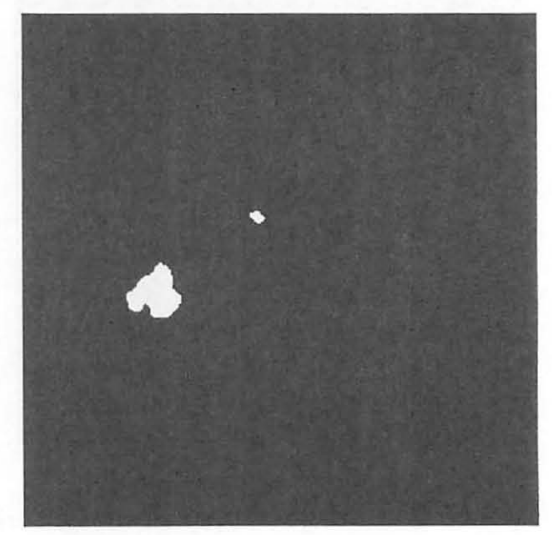

Fig.11 The extractéd region of $\mathrm{HCC}$ 


\section{References}

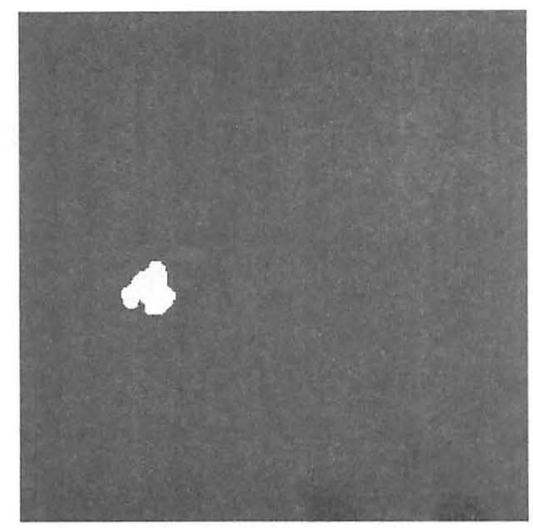

Fig.12 The HCC regions after the post processing

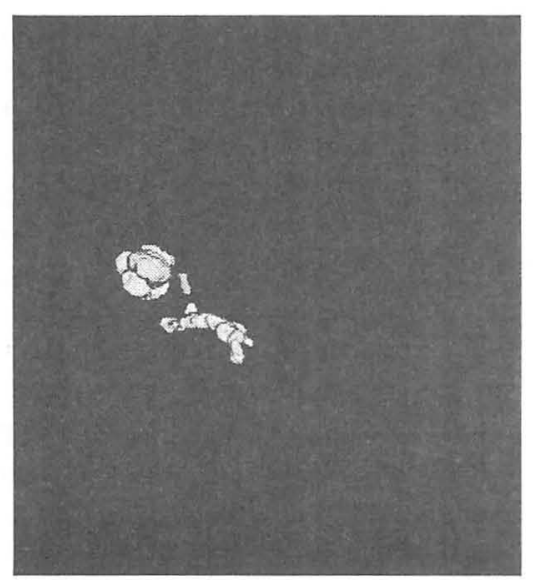

Fig.13 Three-dimensional image of HCC

\section{Conclusions}

In this study, the three-dimensional medical image of the cancer of the liver, which was called HCC, was recognized and extracted by artificial neural network trained using the back propagation algorithm. The regions of $\mathrm{HCC}$ were extracted by the density difference image and the candidate regions of HCC that were obtained from the output image of the neural network. These image processing were carried out for all slices of MDCT images and three-dimensional regions of HCC were displayed clearly.

In the future works, it is needed to eliminate the false positive such as the blood vessels in the liver and apply the medical image processing developed in this study to many liver images.
[1] T. Nagano: Optimization Algorithm, Tokyo, Shokodo, (2000) (in Japanese)

[2] T.Matsuki, T.Kondo, T.Kudo and et.al., Recogniton of 3-dimensional medical images of the lungs, pulmonary vessels and bronchial trees by artificial neural networks, Proceedings of the $39^{\text {th }}$ ISCIE International Symposium on Stochastic System Theory and Its Applications, pp.201-206 (2007)

[3] N. Kodama et.al., Application of Textural Features to the Objective Diagnosis of Alzheimer-type Dementia, Medical Imaging Technology Vol.21 No.1, pp.41-48 (2003) (in Japanese) 